IMMUNOLOGY

\title{
High-salt diet aggravates pyelonephritis
}

\section{$4 c$ \\ Systemic \\ hypergluco- corticoidism induced by an \\ HSD might impair the bactericidal function of neutrophils}

.

Extracellular sodium is known to modulate immune cell function but the effects of a high-salt diet (HSD) on immunity might depend on organspecific responses to high dietary salt. The kidney response to an HSD, for example, might lead to impairment of neutrophil-mediated bacterial clearance, according to a new study by Christian Kurts and colleagues.

"Accumulation of sodium in the skin in response to an HSD enhances macrophage-mediated protection against leishmaniasis," notes Kurts. "The kidney can also accumulate salt, for example in conditions of low fluid intake, and we wanted to test if an HSD might improve immunity against pyelonephritis." By contrast, the researchers found that animals fed an HSD accumulated higher numbers of uropathogenic Escherichia coli (UPEC) in the kidney than those fed a normal-salt diet (NSD). "Most immunological parameters were unchanged by the HSD but the ability of neutrophils to kill phagocytosed bacteria was impaired," explains Kurts. "Neutrophils are crucial to antibacterial immunity, including in pyelonephritis". In vitro, high sodium conditions enhanced the bactericidal function of macrophages but not of neutrophils.

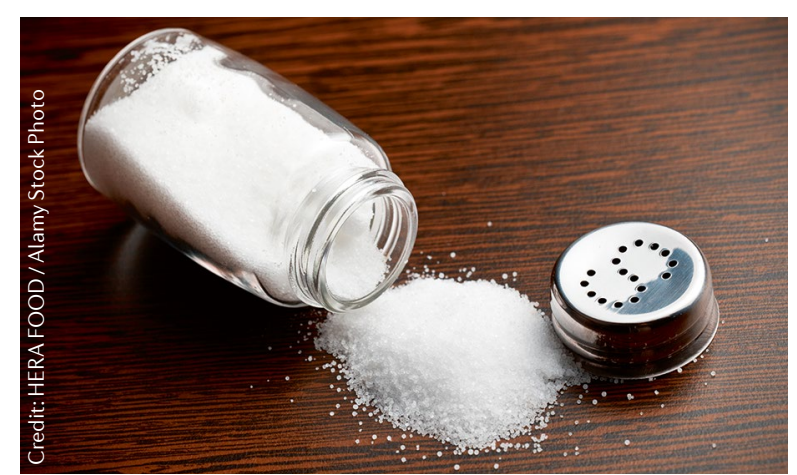

In contrast to the skin, where an HSD induces accumulation of sodium, in the kidney, an HSD led to a rise in intrarenal urea, but not sodium; medullary sodium levels were higher than those in the cortex, as observed under homeostatic conditions. Sodium-induced hyperosmolarity is thought to have a proinflammatory effect that is mediated by the transcription factor nuclear factor of activated T cells 5 (NFAT5). However, despite the unchanged physiological differences in local sodium, an HSD increased Nfat5 levels in both the renal cortex and the medulla, whereas levels of tumour necrosis factor (TNF) and the ratio of intrarenal $\mathrm{T}$ helper 17 cells to regulatory $\mathrm{T}$ cells were unchanged. Moreover, lowering $N f a t 5$ to normal levels with antisense oligonucleotides did not improve pyelonephritis in HSD-fed mice. "These findings suggested that an alternative immunoregulatory factor might be involved," notes Kurts.

RNA sequencing of neutrophils from mice fed an HSD or an NSD revealed that high salt upregulated the expression of glucocorticoid target genes, and the researchers confirmed that an HSD induced hyperglucocorticoidism in mice. This effect might be due to suppression of aldosterone synthesis. Under low-salt conditions, angiotensin II (AngII)-induced conversion of corticosterone into aldosterone leads to changes in the expression of renal sodium transporters and facilitates sodium reabsorption in the kidney. In HSD-fed mice, the researchers observed an accumulation of corticosterone, which could be rescued by an AngII infusion. "Previous studies had indicated that an HSD increases intrarenal urea and systemic glucocorticoids, but the immunological consequences of these effects were unknown," explains Kurts.

Glucocorticoids act as immunosuppressants and dexamethasone decreased the ability of neutrophils, but not macrophages, to internalize phagocytosed bacteria in vitro. In NSD-fed mice, dexamethasone injection exacerbated pyelonephritis, and neutrophils were less able to kill internalized bacteria compared with controls. Moreover, in HSD-fed mice, the glucocorticoid receptor inhibitor mifepristone enhanced the ability of neutrophils to kill UPEC compared with untreated controls. "Systemic hyperglucocorticoidism induced by an HSD might impair the bactericidal function of neutrophils," remarks Kurts. The researchers demonstrated this functional impairment by exposing mice to Listeria monocytogenes - neutrophil clearance of phagocytosed bacteria was reduced in HSD-fed mice compared with those fed an NSD and this effect was rescued by mifepristone.

In healthy humans, consuming an additional $6 \mathrm{~g}$ of salt for 1 week lowered plasma aldosterone, increased plasma corticosterone and impaired the ability of neutrophils to kill UPEC ex vivo.

"In response to an HSD the skin stores sodium but this doesn't happen in the kidney, which instead accumulates urea and increases glucocorticoid levels by blocking aldosterone production in the adrenal gland," explains Kurts. "Our study suggests that the HSD-induced increase in NFAT5 might be due to a systemic rise in glucocorticoids - the links between NFAT5, sodium sensing and immune stimulation may need to be re-evaluated".

Monica Wang

ORIGINAL ARTICLE Jobin, K. et al. A high-salt diet compromises antibacterial neutrophil responses through hormonal perturbation. Sci. Transl. Med.12, eaay3850 (2020) 